\title{
Sustained Effects of Adaptation on the Perception of Familiar Faces
}

\author{
Claus-Christian Carbon \\ University of Bamberg
}

\author{
Thomas Ditye \\ University College London
}

\begin{abstract}
Figural aftereffects are commonly believed to be transient and to fade away in the course of milliseconds. We tested face aftereffects using familiar faces and found sustained effects lasting up to 1 week. In 3 experiments, participants were first exposed to distorted pictures of famous persons and then had to select the veridical face in a 2-alternative forced choice task. Veridicality aftereffects were found in a direction opposite to the adapting distortion; these effects generalized to other pictures of the same individual and also to pictures of celebrities that had not been shown during adaptation. The findings support hierarchical theories of norm-based face coding and suggest that face adaptation effects have a representational basis. They also point toward multiple timescales in the operation of adaptation mechanisms, thereby providing a link between high-level adaptation and more general aspects of neuro-cognitive plasticity, that is, learning and memory.
\end{abstract}

Keywords: face adaptation, face representation, figural aftereffects, familiar faces, plasticity

The impression that the surrounding world is relatively stable and steady arises from constant adjustments of the sensory systems to the ongoing changes in the perceptual environment-a process commonly referred to as adaptation. Adaptation occurs across sense modalities and over wide-ranging time spans (from milliseconds to evolutionary spans; Webster, Werner, \& Field, 2005) as an automatic process that usually remains unnoticed. Experimental adaptation procedures can be used to induce significant misperceptions in the form of figural aftereffects, whose specificity, emergence, duration, and decay provide valuable information about the functional flexibility of the visual system and the higher cognitive processes involved in learning and memory. In the present study, we show that prolonged and continued exposure to distorted face stimuli for approximately $25 \mathrm{~min}$ has sustained effects on the perception of familiar faces ${ }^{1}$ for at least 1 week. These long-term face veridicality aftereffects also transferred across different pictures of the same person as well as across identities. We therefore argue in favor of a representational rather than merely perceptual basis of higher level face aftereffects, with the alteration of a prototype as a possible explanation.

\section{Figural Aftereffects}

Exposure to stimuli that deviate from what is commonly perceived as "normal" alters the perception of subsequently presented

This article was published Online First August 23, 2010.

Claus-Christian Carbon, Department of General Psychology and Methodology, University of Bamberg; and Thomas Ditye, Institute of Cognitive Neuroscience, University College London.

We thank Tilo Strobach for the preparation of the stimuli that were used in this study. We also thank Christian Schmidt and Joel Kuntonen for proofreading the manuscript. We are grateful to Isabel Gauthier, Rachel Robbins, and two anonymous reviewers for their very helpful comments on an earlier version of the manuscript.

Correspondence concerning this article should be addressed to Thomas Ditye, Institute of Cognitive Neuroscience, University College London, 17 Queen Square, London, WC1N 3AR, England. E-mail: thomas.ditye@ ucl.ac.uk material in respect of the manipulated stimulus property. For instance, after exposure to a line that is strongly tilted to the right, a vertical line appears to be slightly tilted to the left (tilt aftereffect; Gibson \& Radner, 1937). Similar normalization-based aftereffects have been found for many basic stimulus attributes (for a historical review on low-level aftereffects, see Wade \& Verstraten, 2005). Aftereffects show inter ocular transfer (Wade, Swanston, \& Deweert, 1993) and invariance to size and position (Suzuki \& Cavanagh, 1998). Thus, it is relatively clear that, unlike their phenomenological relatives, afterimages, aftereffects cannot solely be explained by the adjustment of receptors in the retina. In fact, the site of adaptation has been attributed to neurons in the early regions of the visual cortex thereby assuming a close relationship

\footnotetext{
${ }^{1}$ In the context of this study, familiar faces are defined as faces that the participants were familiar with (i.e., for which they had well-established mental representations) before the beginning of the experiment as opposed to unfamiliar faces that are new to the participants. In most face adaptation studies, unfamiliar faces are usually learned during the initial phase of the experimental procedure. Within the class of familiar faces, an important distinction has to be made between famous faces (the faces of celebrities and public figures) and personally familiar faces (the faces of family members, friends, associates, etc.). Famous faces are usually encountered in specific settings and contexts (i.e., the media), while personally familiar faces are likely to be seen in a wider variety of contexts, and perhaps more frequently; thus individual famous faces may show less variation between instances compared to personally familiar faces. Accordingly, faces in these two classes might be processed and represented in different ways. Carbon (2008) found that students were able to correctly identify pictures of university lecturers (personally familiar face pool) despite small- to medium-large manipulations of their facial appearance, while recognition performance for famous faces known from the media (famous face pool) broke down when the faces were manipulated. This suggests that famous faces might be represented as icons or celebrity snapshots rather than as faces per se. Taylor et al. (2009) showed in a brain imaging study that brain activation induced by personally familiar faces was more widespread and bilaterally distributed, while famous faces elicited activity lateralized to the right hemisphere in the same areas as unfamiliar faces.
} 
between the specific low-level stimulus properties and featureselective neurons in the corresponding cortical areas, for example, orientation-specific neurons in visual area V1 as a basis for the tilt aftereffect. However, as Leopold and Bondar (2005) pointed out, adaptation to any visual stimulus is likely to affect neurons in not only the early stages but also the intermediate and late stages of the visual pathway, all of which may contribute to the aftereffect in one way or another. Such higher level contributions are also suggested by more recent findings in adaptation research based on the use of more complex stimuli, for example, natural images (Webster \& Miyahara, 1997), artworks (Carbon, Ditye, \& Leder, 2007; Carbon \& Leder, 2006), body parts (Kovacs et al., 2006), and not least, faces (e.g., Carbon, Strobach, et al., 2007; Leopold, O'Toole, Vetter, \& Blanz, 2001; Webster \& MacLin, 1999). Faces are objects of tremendous social importance whose stimulus dimensions are subject to higher cognitive processing and usually cannot be found (or tested) in simpler visual materials (e.g., identity, emotional expression, attractiveness). Studying adaptation in the face domain is of high relevance to our understanding of both the fundamental neural and cognitive principles of higher level aftereffects and the perception of faces as well as the way in which they are represented and flexibly updated in memory.

\section{Face Adaptation}

Webster and MacLin (1999) were the first to show that adaptation to a distorted picture of a face leads to a bias in the perception of the original face in a direction opposite to the adapting distortion (face distortion aftereffect). That means, for instance, that following adaptation to a configurally extended face, the original face appears compressed (and thus not veridical), and vice versa. Since then, a significant number of different face aftereffects have been identified. In the face identity aftereffect, exposure to a particular face distorts the perceived identity of another face that is presented subsequent to adaptation (Leopold et al., 2001; Leopold, Rhodes, Muller, \& Jeffery, 2005; Rhodes \& Jeffery, 2006). Rhodes, Jeffery, Watson, Clifford, and Nakayama (2003) applied an adaptation paradigm to alter participants' perception of what constituted a "normal face" and showed that these alterations also were accompanied by increased attractiveness ratings for faces more similar to the new norm. Following this approach, aftereffects for natural facial attributes have been determined also to include gender, ethnicity, facial expression (e.g., Webster, Kaping, Mizokami, \& Duhamel, 2004), and eye gaze (Jenkins, Beaver, \& Calder, 2006; Schweinberger, Kloth, \& Jenkins, 2007) aftereffects. These findings are very robust and similar effects could also be demonstrated in highly familiar faces (Carbon \& Leder, 2005, 2006; Carbon, Strobach, et al., 2007), whose recognition is believed to depend on particularly stable representations (e.g., Bruce, 1994). Moreover, face aftereffects generalize to images of various size (Rhodes et al., 2004; Zhao \& Chubb, 2001), are partly invariant to position (Kovacs, Cziraki, Vidnyanszky, Schweinberger, \& Greenlee, 2008; Kovacs, Zimmer, Harza, \& Vidnyanszky, 2007), are not selective for color, contrast, or viewpoint (Jiang, Blanz, \& O'Toole, 2006; Yamashita, Hardy, De Valois, \& Webster, 2005) and transfer across different identities (Carbon, Strobach, et al., 2007; Robbins, McKone, \& Edwards, 2007; Webster \& MacLin, 1999; Yamashita et al., 2005). The ability to generalize across stimulus attributes indicates that higher level neural mechanisms lead to an alteration of the mental representation of faces and cause a postadaptation mismatch between the test stimulus and the corresponding representation that is stored, and was updated, in memory.

The aftereffects reviewed above can be explained in the framework of norm-based theories of face coding. Besides other (e.g., exemplar-based) models (Valentine \& Endo, 1992), it has been proposed that individual faces might be represented by their deviations from an average face that is abstracted from all faces ever encountered (Valentine, 1991, 2001). This prototype, norm, or average face is thought to serve as a reference point for all individual faces in the face space. According to this line of thought, it has been argued that the adaptation procedure is likely to alter (one or multiple) face norms along the manipulated feature dimension (e.g., identity, gender, ethnicity, etc.) toward the adapting stimuli. This results in a change of distance between the new, adapted face norm and individual exemplars. An increase in distance therefore causes the respective individual face to be perceived less typical than before whereas a decrease in distance leads to a more normal appearance (Carbon \& Leder, 2005, 2006; Carbon, Strobach, et al., 2007; Hurlbert, 2001; Leopold et al., 2001; 2005; Rhodes \& Jeffery, 2006; Webster et al., 2004; Webster \& MacLin, 1999). Extending this idea, several norms could exist, reflecting different hierarchical levels of face representations, from an individual norm, against which single pictures of a specific individual are coded and norms for particular face subpopulations (e.g., male or female faces, faces of a specific ethnic group, etc.) to a single generic norm that is the average of all faces and that encodes the representation of faces as an object class (Rakover, 2002; Rhodes, Brennan, \& Carey, 1987; Rhodes et al., 2005). Adaptation transfer effects are of particular interest as they shed light not only on feature selectivity but also on the organizational principles of multiple face representations in the perceptual system. In studies investigating contingent aftereffects, it has been demonstrated that the transfer of aftereffects is more pronounced when adaptation and test stimuli are matched on specific dimensions (e.g., gender; Bestelmeyer et al., 2008; Jaquet \& Rhodes, 2008; Little, DeBruine, \& Jones, 2005), which points toward feature-selective neurons in high-level vision. Jiang, Blanz, and O'Toole (2009) recently found that learning multiple face views enhanced the transfer of adaptation effects across illumination changes and thus suggested that three-dimensional information is available in representations of familiar faces. In addition, Carbon, Strobach, et al. (2007) were able to show that face veridicality aftereffects in highly familiar faces transferred to other pictures of the same person, but not to pictures of other individuals, suggesting identity-specific face representations that operate independently from more general face norms. The study reported in this paper followed this approach and focused primarily on the temporal duration of such cross-prototype transfer effects in face adaptation. To this end, the study employed a prolonged adaptation procedure designed to maximize adaptation time while maintaining the participants' interest.

\section{The Time Course of Face Aftereffects}

Adaptation effects are primarily influenced by three temporal variables: (1) adaptation duration, the time span during which the adapting stimulus is presented; (2) delay, the time span between 
adaptation and test; and (3) test duration, the time span during which the test stimulus is presented. In the studies reviewed above, adaptation duration ranged from $5 \mathrm{~s}$ to 5 min (Leopold et al., 2001; Rhodes et al., 2003; Webster \& MacLin, 1999) and test duration varied somewhat unsystematically from $200 \mathrm{~ms}$ to unlimited (Carbon \& Leder, 2005; Leopold et al., 2001; Webster et al., 2004; see Table 1 for a summary).

Rhodes, Jeffery, Clifford, and Leopold (2007) counterbalanced adaptation duration (1,000 to $16,000 \mathrm{~ms}$ ) and test duration (200 to $3,200 \mathrm{~ms}$ ) and found that figural and identity aftereffects increased logarithmically with adaptation duration and decreased exponentially with test duration. This pattern matches the one found in previous studies on the time course of face (Leopold et al., 2005) and traditional aftereffects (e.g., Hershenson, 1989; Krauskopf, 1954), indicating that the dynamics of high-level aftereffects are similar to those of low-level aftereffects. Rhodes et al. (2007) concluded that visual adaptation seems to rely on similar processes throughout the visual system. Much less research exists on the temporal delay between adaptation and test, which is surprising, as it would provide useful information about the decay of aftereffects and thus the "recalibration" and "re-adaptation" abilities of the visual system. Such research also might provide important information about the quality of adaptation processes, namely as to whether they are based more on perception or representation.

It is commonly believed that face aftereffects "die away in less than a second" (Hurlbert, 2001, p. 4), and early studies of simple aftereffects arrived at the same conclusion (Bales \& Follansbee, 1935; Hammer, 1949). However, there are also indications for longer lasting aftereffects. Vernon (1934) found aftereffects in the perception of tilted lines 5 min after adaptation, Koehler (1944) reported on a single subject in which the tilt aftereffect persisted for at least $24 \mathrm{hr}$ and Neitz, Carroll, Yamauchi, Neitz, and Williams (2002) measured shifts in color perception that persisted for "one to two" weeks (p. 783). In the motion domain, it has been shown that longer adaptation durations, such as 15 min of adaptation to a moving spiral pattern (Masland, 1969) and up to $4 \mathrm{hr}$ of adaptation to a moving color pattern (Hepler, 1968), can induce negative aftereffects lasting for at least $20 \mathrm{hr}$. Moreover, the McCollough effect (McCollough, 1965)—an orientationcontingent color aftereffect in which adaptation to, for example, blue vertical and yellow horizontal gratings makes subsequently presented neutral gratings appear yellowish and bluish, respectively-persisted for as long as 85 days when participants were tested only once as opposed to repeated testing during the intermediate time period (Jones \& Holding, 1975). These results are supported by a more recent study by Vul, Krizay, and MacLeod (2008) on the McCollough effect that provides convincing evidence that adaptation processes operate simultaneously on multiple timescales. The findings suggest at least two distinct and separable timescales that seem to work independently and in parallel: A fast timescale that gives rise to strong aftereffects that saturate after about $30 \mathrm{~s}$ and decay quickly, and a slow, infinite timescale that shows no signs of decay. The latter is referred to as a perfect integrator in terms of a permanent shift in baseline. The magnitude of the baseline shift did not saturate, but increased linearly with adaptation duration, pointing toward an integrator that "remembers all of the stimuli that were ever presented" (p. 4), a concept closely related to the normalization and prototype-based mechanisms reviewed above.

Recent findings suggest that long-term processes also operate in face adaptation. Kloth and Schweinberger (2008) reported on eye gaze aftereffects that decayed in the course of $7 \mathrm{~min}$. McKone, Edwards, Robbins, and Anderson (2005) were able to show that aftereffects induced by $160 \mathrm{~s}$ of adaptation to radially as well as featurally compressed or extended faces survived a 15-min delay even if normal undistorted faces were presented between adaptation and test. At testing after $24 \mathrm{hr}$ the effect was gone. For familiar faces, long-term aftereffects were found for a period of $5 \mathrm{~min}$ and (to a lesser degree but still reliable) $24 \mathrm{hr}$ (Carbon, Strobach, et al., 2007). These findings are puzzling in some respects, as one would expect the neural networks to readapt quickly to the natural configurations of the visual environment, especially in cross-session designs. On the other hand, in the case of immediate aftereffects, it is legitimate to ask why changes to the representation of visual

Table 1

Combinations of Adaptation Duration, Delay, and Test Duration in Selective Studies on Face Adaptation in Chronological Order

\begin{tabular}{|c|c|c|c|}
\hline Study & Adaptation duration & Delay & Test duration \\
\hline Webster \& MacLin (1999) & $5 \min (+8 \mathrm{~s}$ top-up) & NA & $1 \mathrm{~s}$ \\
\hline Leopold, O'Toole, Vetter, \& Blanz (2001) & $5 \mathrm{~s}$ & $150,300,600,1,200,2,400 \mathrm{~ms}$ & $200 \mathrm{~ms}$ \\
\hline Rhodes, Jeffery, Watson, Clifford, \& & & & \\
\hline $\begin{array}{l}\text { Nakayama (2003) } \\
\text { Webster, Kaping, Mizokami, \& Duhamel }\end{array}$ & $5 \min (+8 \mathrm{~s}$ top-up) & $500 \mathrm{~ms}$ & $1,500 \mathrm{~ms}$ \\
\hline (2004) & $180 \mathrm{~ms}(+5 \mathrm{~s}$ top-up) & $250 \mathrm{~ms}$ & Unlimited \\
\hline Carbon \& Leder (2005) & $30 \mathrm{~s}$ & $4 \mathrm{~s}, 5 \mathrm{~min}$ & Unlimited \\
\hline Leopold, Rhodes, Muller, \& Jeffery (2005) & $1,2,4,8,16 \mathrm{~s}$ & - & $100,200,400,800,1,600 \mathrm{~ms}$ \\
\hline $\begin{array}{l}\text { McKone, Edwards, Robbins, \& Anderson } \\
(2005)\end{array}$ & $160 \mathrm{~s}$ & $15 \mathrm{~min}$ & NA \\
\hline Carbon and Leder (2006) & $30 \mathrm{~s}$ & $80 \mathrm{~min}$ & NA \\
\hline Carbon, Strobach, et al. (2007) & $\sim 36 \mathrm{~min}$ & $5 \mathrm{~min}, 24$ hours & Unlimited \\
\hline Rhodes, Jeffery, Clifford, \& Leopold (2007) & $1,2,4,8,16 \mathrm{~s}$ & $1,000 \mathrm{~ms}$ & $200,400,800,1,600,3,200 \mathrm{~ms}$ \\
\hline $\begin{array}{l}\text { Kovacs, Zimmer, Harza, \& Vidnyanszky } \\
\text { (2007) }\end{array}$ & $500 \mathrm{~ms}, 5 \mathrm{~s}$ & $500 \mathrm{~ms}$ & $200 \mathrm{~ms}$ \\
\hline Kloth \& Schweinberger (2008) & $\begin{array}{l}1 \text { min, } 24 \mathrm{~s}(+3.5 \mathrm{~s} \text { top-up in } \\
\text { the first test block) }\end{array}$ & 0 to $10 \mathrm{~min}$ & $400 \mathrm{~ms}$ \\
\hline
\end{tabular}

Note. $\quad$ Delay $=$ interval between adaptation and test; top-up $=$ additional adaptation stimulus preceding each test trial; NA $=$ not available. 
stimuli in the form of a normalization process would passively decay at all if no re-adaptation period had an opportunity to take effect. Adaptation duration could therefore be a key variable for the investigation of the neuro-cognitive mechanisms underlying short- and long-term face adaptation. In the present study, we used a prolonged adaptation procedure $(\sim 25 \mathrm{~min})$ to induce strong and long-lasting aftereffects in the perception of familiar faces and then measured them $5 \mathrm{~min}$ (Experiment 1), $24 \mathrm{hr}$ (Experiment 3), and 1 week (Experiment 2) after adaptation.

\section{The Present Study}

Three experiments are reported in which participants were exposed to configurally compressed or extended versions of highly familiar faces and later asked to select the veridical face from two alternative stimuli (the original and slightly compressed/extended versions of the faces). We expected that adaptation to strongly distorted faces would alter participants' representations of the respective individual faces as well as general face representations toward the adaptation stimulus. Following adaptation, the original versions would therefore appear distorted in a direction opposite to the adapting distortion (e.g., the face would appear compressed if the participant had adapted to extended faces, and vice versa). When the participants were asked to select the veridical face, we consequently expected them to pick a slightly distorted version of the face (the one that was more similar to the adapting stimuli) when it was presented together with the original. In the first experiment, we investigated face veridicality aftereffects in highly familiar faces with a 5-min delay between adaptation and test. The generalization of aftereffects was tested by using not only the same pictures for adaptation and test (condition picture), but also alternative pictures of the same individual (identity) and pictures of persons that were not shown during adaptation (novel). This structure is similar to that of the experiments reported by Carbon, Strobach, et al. (2007), with the difference that a new test procedure was introduced. Instead of morphing the test pictures on a distortion continuum to select the veridical face (Carbon, Strobach, et al., 2007), which was a problematic procedure due to the continuous adaptation processes taking place during the selection process (for details, refer to the discussion of Carbon, Strobach, et al., 2007), we used an alternative experimental approach. In the present study, participants were asked to select one of two versions of each test face (the original and a slightly distorted version) that were presented simultaneously (two-alternative forced choice [2AFC]). The focus of Experiment 2 was on the long-term effects of adaptation and thus the delay between adaptation and test was extended to 1 week. Experiment 3 was a follow-up experiment that intended to rule out potential confounding factors (i.e., context effects) that otherwise would have provided an alternative explanation for the significant transfer effect found in the previous experiments. In this experiment, cross-identity transfer effects were tested separately by omitting the celebrity faces used in the adaptation phase from the test task.

\section{Experiment 1}

\section{Method}

Participants. Thirty-six undergraduate students (30 women; $M$ age: 22.9 years, range: 19 to 38 ) from the University of Vienna,
Austria, took part to fulfill course requirements. Participants were naïve to the purpose of the experiment and had normal or corrected-to-normal vision, as assessed by standard vision tests.

Apparatus and stimuli. Two different frontal-view pictures (Images A and B) of 27 celebrities, who are well-known to the German-speaking public, were selected as familiar faces. ${ }^{2}$ The distances between the eyes and the mouth in each of the pictures were gradually compressed and extended, resulting in 11 different versions of each picture (five compressed versions, five extended plus the original). Distortion intervals were two pixels for Versions -1 to -4 (or +1 to +4 , respectively); another step of 10 pixels was added in Versions -5 and +5 to create particularly large distortions. Similar techniques have been used in other face adaptation or priming studies (Carbon \& Leder, 2005, 2006; Carbon, Strobach, et al., 2007; Faulkner, Rhodes, Palermo, Pellicano, \& Ferguson, 2002; Robbins et al., 2007); see Figure 1A for an illustration. Each celebrity was randomly allocated to one of three stimulus sets corresponding to the three levels of adaptation transfer: picture, identity, and novel. Each set included nine celebrities. The approximate size of the pictures was $220 \times 240$ pixels and the experiment was controlled by the experimental software PsyScope PPC 1.25 (Cohen, MacWhinney, Flatt, \& Provost, 1993), which was run on a Macintosh (OS 9.2.2) eMac with a 17“" monitor at a resolution of $1,024 \times 768$ pixels.

Procedure. The experiment consisted of three phases: adaptation, delay, and test.

Adaptation phase. During adaptation, participants were either exposed to the strongly compressed versions ( -5 , see Figure 1A), the original versions $(0)$, or the strongly extended versions $(+5)$ of celebrity faces from two stimulus sets (between-subjects factor distortion). Participants were exposed either to Image A or Image $\mathrm{B}$ of a particular individual. The pictures assigned to the third stimulus set were not shown during adaptation, and both image and set were counterbalanced across participants. Each picture was presented 15 times. Five different screen positions were used to control for potential effects of retinal position and to make the task more interesting. These positions were crossed with three different presentation durations: 2, 3, and $4 \mathrm{~s}$. Position and duration were both randomized. Stimulus presentation was preceded by a sequence starting with a fixation cross $(500 \mathrm{~ms})$ in the center of the target position, followed by a rectangular frame $(200 \mathrm{~ms})$ used to guide participants' attention. After each stimulus presentation, participants were requested to judge whether the previous stimulus was a picture of a male or a female face. They indicated their selections by pressing the corresponding button on a keyboard. The gender-decision task was time sensitive in that participants were asked to make their decision as quickly as possible after the stimulus disappeared. Together with the variable presentation time, this task was designed to help keep participants in a state of constant alertness. Each response was followed by the feedback

\footnotetext{
${ }^{2}$ Male: Franz Beckenbauer, Boris Becker, Pierce Brosnan, George W. Bush, Nicholas Cage, Bill Clinton, George Clooney, Tom Cruise, Thomas Gottschalk, Oliver Kahn, Helmut Kohl, Jack Nicholson, Brad Pitt, Gerhard Schröder, Michael Schumacher, Arnold Schwarzenegger, Rudi Völler; Female: Pamela Anderson, Cindy Crawford, Princess Diana, Cameron Diaz, Verona Feldbusch, Nicole Kidman, Madonna, Angela Merkel, Julia Roberts, Claudia Schiffer.
} 
A

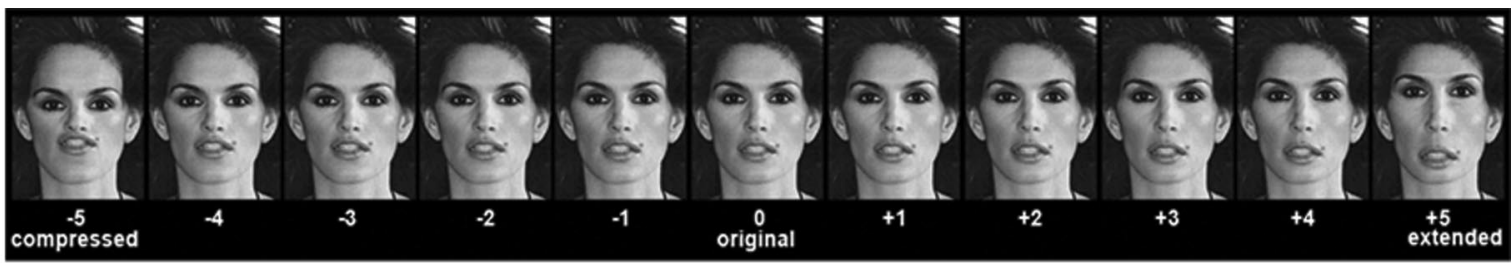

B

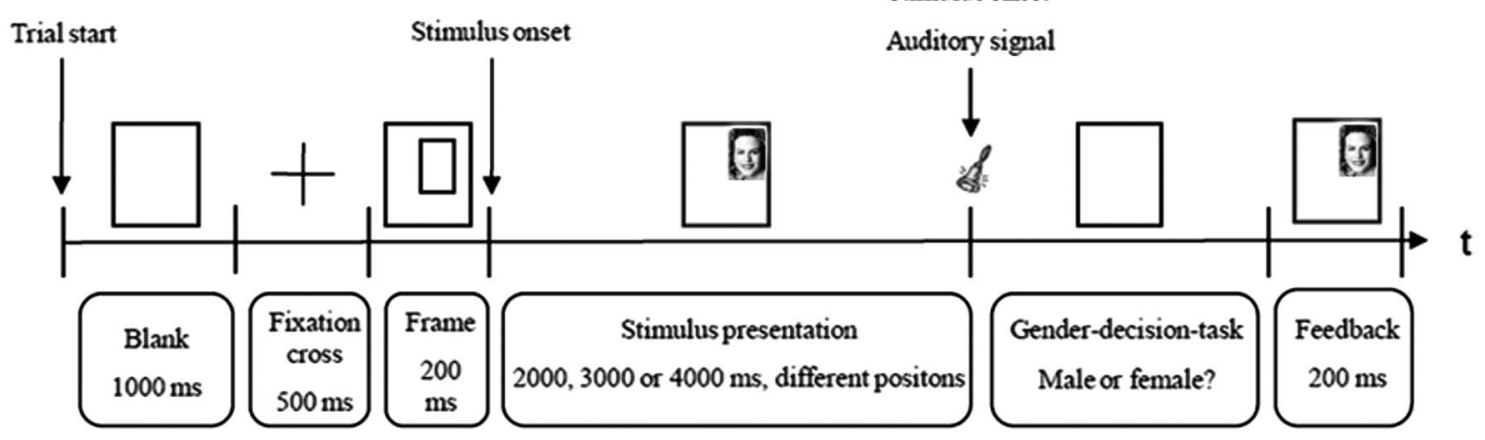

C

Transfer of the adaptation-effect

Adaptationphase

Test phase

\begin{tabular}{ccc} 
Picture & Identity & Novel \\
\hline & & \\
\hline & &
\end{tabular}

Figure 1. Design and stimuli. (A) The original picture of Cindy Crawford (middle), five gradually compressed versions $(-1$ to -5$)$ and five gradually extended versions $(+1$ to +5$)$ of her face. (B) Prolonged adaptation procedure. (C) Three conditions were used to examine whether the adaptation effect is limited to pictorial information (picture) or also generalizes to different pictures of the same person (identity) and/or to completely new familiar faces (novel). $\mathrm{t}=$ time.

presentation of the picture for $200 \mathrm{~ms}$ to maximize inspection time. This prolonged adaptation procedure consisted of 18 [persons] $\times$ 15 [repetitions] $=270$ trials and took approximately $25 \mathrm{~min}$ (Figure 1B).

Delay phase. Adaptation and test were separated by a 5-min delay during which participants engaged in informal conversation with the experimenter.
Test phase. After the delay, the test was conducted. In a $2 \mathrm{AFC}$ task, participants had to select the veridical face out of two versions of each face. Following the original instructions of Carbon, Strobach, et al. (2007), we explicitly instructed the participants to base their decisions on their world knowledge (images known from the media), and not on any experimentally seen images. The instructions were: "Which is the veridical version of 
the face?" In each trial, either the slightly compressed version (i.e., the -2 version on the distortion scale; see Figure 1A) or the slightly extended version $(+2)$ was presented together with the original. Each picture was shown four times with the various versions appearing in different, randomized locations (original left/ compressed right; original right/compressed left; original left/ extended right; original right/extended left). To test the generalization of possible adaptation effects, the within-subject factor transfer was manipulated on three levels (Figure 1C). In the case of the celebrities included in the first stimulus set, participants were tested with exactly the same picture that was used for adaptation (picture). For the celebrities in the second stimulus set, the alternative image of the same individual was used for testing (identity), and the test phase also included one image (A or B) of each celebrity from the remaining stimulus set that had not been shown during adaptation (novel).

Finally, the participants were asked to rate all pictures according to familiarity (whether they knew the depicted celebrities from the media) and typicality (the extent to which the pictures used in the experiment were typical of the depicted celebrities). As face veridicality can be assessed only when the target face is actually known, these ratings were used to exclude trials that involved faces unfamiliar to the participant.

\section{Results and Discussion}

On average, participants indicated their familiarity with $94.8 \%$ of the celebrities, with a range from $72.2 \%$ to $100 \%$. Trials including celebrities that were unfamiliar to a participant were excluded from further analyses of that participant's data, even if only one of the two images (A or B) was not recognized. As a result, $7.2 \%$ of the collected data were excluded. Selected test face served as the dependent variable and was the mean of multiple-test face selections with the compressed versions, which were coded -2 in accordance with the extent of deviation measured in pixels on the distortion continuum (see Figure 1A); the original versions were coded 0 and the extended versions +2 .

A two-way mixed-design analysis of variance (ANOVA) was calculated with the between-subjects factor distortion (adaptation faces -5 , original, and +5 ) and the within-subject factor transfer (levels picture, identity, novel). There was a significant main effect for distortion, $F(2,33)=52.01, p<.001, \eta_{\mathrm{p}}^{2}=.76$, with $M_{-5}=$ $-.40, M_{\text {original }}=.08$, and $M_{+5}=.41$. Bonferroni-corrected post hoc comparisons revealed significant differences between all possible pairs, $p<.001$. This can be interpreted as an overall adaptation effect that lasted for at least $5 \mathrm{~min}$ and indicates that the adaptation procedure biased participants' perception of the test stimuli as predicted. The main effect of transfer was not significant, $F(2,66)<1, p=.63, \eta_{\mathrm{p}}^{2}=.14$; however, there was an interaction between distortion and transfer, $F(4,66)=8.01, p<$ $.001, \eta_{\mathrm{p}}^{2}=.33$; see Figure 2. Univariate ANOVAs for the factor distortion revealed highly significant effects for all levels of transfer: picture, $F(2,33)=57.53, p<.001, \eta_{\mathrm{p}}^{2}=.78$; identity, $F(2$, $33)=34.66, p<.001, \eta_{\mathrm{p}}^{2}=.68$; and novel, $F(2,33)=20.67, p<$ $.001, \eta_{\mathrm{p}}^{2}=.56$. In both experimental conditions, simple main effects showed a weaker effect for novel compared to picture $\left(-5: p \mathrm{~s}<.001 ; M_{\text {picture }}=-.52, M_{\text {novel }}=-.29 ;+5: p \mathrm{~s}<.001 ;\right.$ $\left.M_{\text {picture }}=.53, M_{\text {novel }}=.25\right)$. In the +5 condition, novel was also weaker than identity $\left(p \mathrm{~s}=.007 ; M_{\text {identity }}=.45\right)$. We found no
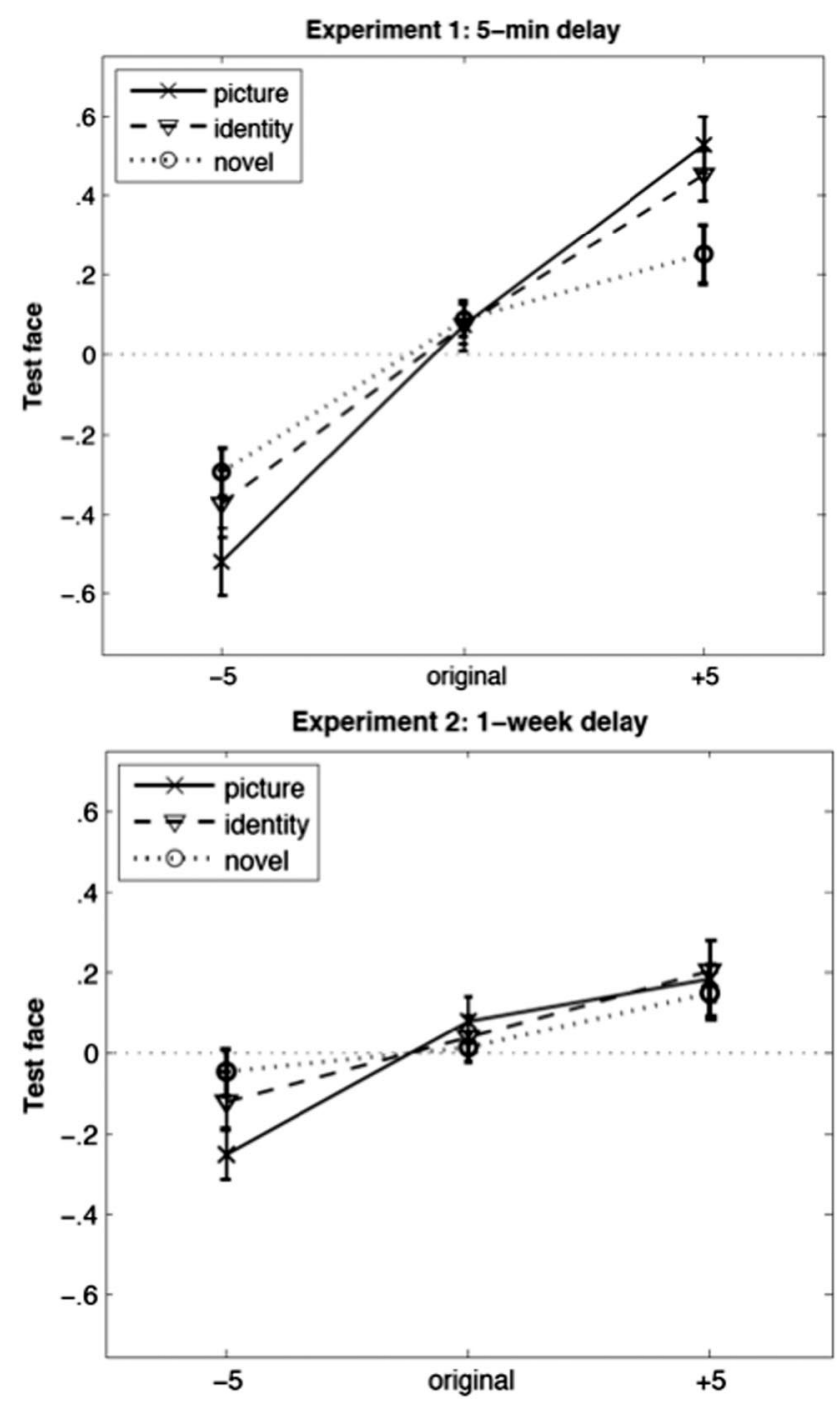

Figure 2. Results. Experiment 1: 5-min delay; Experiment 2: 1-week delay.

significant differences between picture and identity, which favors the idea of an adaptation procedure that operates on the level of individual representations rather than on that of single pictures, suggesting that higher level mechanisms are involved. The novel effect was weaker but still reliable in both experimental conditions, indicating that adaptation affects not only the mental representation of specific individual faces, but also the face space in general. This more abstract type of representation in the sense of broader face subpopulations or the general average face is in line with hierarchical theories of norm-based face coding (Rakover, 2002; Rhodes et al., 2005).

There was a slight but significant shift in the control condition (original) toward the positive array, $M=.08 ; t(35)=2.68, p=$ .011. This might have been caused by an imbalance in the perceived degree of distortion of the -2 and the +2 test faces; the extended faces looked more natural compared to the compressed faces and as a result they were more frequently selected in error. 
To test for the sustained effects of the adaptation procedure on the perception of familiar faces, the delay between adaptation and test was extended to 1 week in Experiment 2.

\section{Experiment 2}

\section{Method}

Participants. Thirty-six undergraduate students (27 women; $M$ age: 23.2 years, range: 19 to 43 ) from the University of Vienna, Austria, took part to fulfill course requirements. Participants were unaware of the purpose of the experiment and had normal or corrected-to-normal vision, as assessed by standard vision tests. None of them had taken part in Experiment 1.

Procedure. The procedure of Experiment 2 was similar to that of Experiment 1, with the difference that the participants left after the adaptation phase and were asked to come back to the lab approximately 1 week later. The mean interval between the two sessions was $168.53 \mathrm{hr}$, which is 7 days and $30 \mathrm{~min}$, with a range from 164 to $169 \mathrm{hr}$. One participant was tested $197 \mathrm{hr}$ (8 days and $5 \mathrm{hr}$ ) after adaptation.

\section{Results and Discussion}

The participants were highly familiar with the celebrities $(M=$ 95.94\%; range: $64.8 \%$ to $100 \%$ ). Trials including faces that the participants rated as unfamiliar were excluded on an individual basis, leading to the elimination of a total of $6.3 \%$ of the data.

The results of a two-way mixed-design ANOVA including the factors distortion and transfer showed a significant main effect of distortion, $F(2,33)=8.74, p=.001, \eta_{\mathrm{p}}^{2}=.350$, with the following means: $M_{-5}=-.14, M_{\text {original }}=.05, M_{+5}=.18$. Bonferroni comparisons revealed a significant difference between -5 and $+5(p=.001)$. The mean of the control group (original) did not differ from either of the experimental groups ( $-5 \mathrm{vs}$. original: $p=.068$; original vs. $+5: p=.257$ ). This can be interpreted as an overall effect of face adaptation that was still found after 1 week.

There was no main effect for transfer, $F(2,33)=0.55, p=.58$, $\eta_{\mathrm{p}}^{2}=.020\left(M_{\text {picture }}=.07, M_{\text {identity }}=.04, M_{\text {novel }}=.04\right)$; however, the interaction between transfer and distortion was significant, $F(4,66)=2.64, p=.04, \eta_{\mathrm{p}}^{2}=.140$; see Figure 2. Simple main effects showed a significant difference between the levels picture and novel in the -5 group $\left(p s=.02, M_{\text {picture }}=-.25, M_{\text {novel }}=\right.$ -.47). The results of three univariate ANOVAs indicated highly significant effects of distortion for picture, $F(2,33)=9.7, p<$ $.001, \eta_{\mathrm{p}}^{2}=.37$, and identity, $F(2,33)<1, p=.008, \eta_{\mathrm{p}}^{2}=.253$, and a just significant effect for novel, $F(2,33)=3.40, p=.046$, $\eta_{\mathrm{p}}^{2}=.171$. Pairwise comparisons showed the following differences: picture: -5 versus original $\left(M_{-5}=-.25, M_{\text {original }}=.08, p=\right.$ $.009)$ and -5 versus $+5\left(M_{-5}=-.25, M_{+5}=.19, p s=.001\right)$; identity: -5 versus $+5\left(M_{-5}=-.12, M_{+5}=.20, p=.006\right)$; novel: -5 versus $+5\left(M_{-5}=-.05, M_{+5}=.152, p=.047\right)$. Adaptation affected participants' perception not only immediately, but also for a much longer time period; this was true for all levels of transfer. It appears that 1 week was not long enough for the perceptual system to recalibrate itself and thus fully recover from the prolonged adaptation that was applied in the previous adaptation phase. The significant 1-week transfer effect is somewhat surprising. We expected it to vanish in the course of several days due to re-adaptation processes as participants were most probably exposed to natural, that is, veridical, face configurations in everyday life. We suspected that this finding might have been influenced by the effects of episodic memory and therefore tested this hypothesis in Experiment 3.

To compare Experiment 2 to Experiment 1, the betweensubjects factor delay with the levels 5 min and 1 week was added into a three-way mixed-design ANOVA. There was no main effect for delay, $F(1,66)=<1, p=.95\left(M_{5 \text { min }}=.03, M_{1 \text { week }}=.03\right)$, but a significant interaction was found between delay and distortion, $F(2,66)=9.90, p<.001, \eta_{\mathrm{p}}^{2}=.230$. As revealed by the simple main effects for each level of distortion, the difference between $5 \mathrm{~min}$ and 1 week was significant in both experimental conditions $(-5: p=.002 ;+5: p=.004)$, but not in the control condition ( $p=.66)$, indicating a significant overall decline in the size of the adaptation effects over time. The three-way interaction Distortion $\times$ Transfer $\times$ Delay was not significant, $F(4,132)=$ $1.91, p=.11, n s$, which indicates that the decay rates for the picture, identity, and novel conditions are similar.

\section{Experiment 3}

The aim of Experiment 3 was to control for a possible confounding factor that might have caused the significant long-term transfer effect found in Experiment 2 (i.e., the effects of episodic memory). We suspected that episodic memories of the adaptation session had biased participants' selections at test toward specific earlier experiences. To control this variable, we intended to dissociate participants' experiences and thereby reduce the first session's impact on the second one. Hence in Experiment 3 the pictures used during adaptation were completely omitted from the test phase. This procedure allowed us to isolate the transfer condition and test it separately from the other conditions.

\section{Method}

Participants. Eighteen undergraduate students (15 women; $M$ age: 22.1 years, range: 19 to 32 ) from the University of Vienna, Austria, took part to fulfill course requirements. All participants had normal or corrected-to-normal vision, as assessed by standard vision tests, and were naïve to the purpose of the experiment. None of them had taken part in either of the previous experiments.

Procedure. The general procedure of Experiment 3 was similar to that of Experiments 1 and 2; however, at test the conditions picture and identity were omitted and only the pictures from the novel stimulus set were presented. The delay was $24 \mathrm{hr}$. It was assumed that this delay would be long enough to investigate long-term adaptation effects and also short enough to ensure that the transfer effect-in case it existed-would still be present. The mean interval between adaptation and test was $24 \mathrm{hr}$ and $12 \mathrm{~min}$, with a range from $21 \mathrm{hr}$ and $26 \mathrm{~min}$ to $26 \mathrm{hr}$.

\section{Results and Discussion}

Participants' average familiarity with the celebrities was $94.24 \%$, ranging from $79.63 \%$ to $100 \%$, and $4.8 \%$ of the total data were excluded for reasons of unfamiliarity. The results of a univariate ANOVA with the between-subjects factor distortion $(-5$, 
original, +5 ) showed a considerable adaptation effect (i.e., transfer effect), $F(2,15)=15.33, p<.001, \eta_{\mathrm{p}}^{2}=.67$, with the following means: $M_{-5}=-.27, M_{\text {original }}=-.1, M_{+5}=.31$ (see Table 2). Bonferroni comparisons indicated significant differences between the groups -5 and $+5(p<.001)$ and original and $+5(p=.006)$. There was a significant shift of the mean of the control group toward the negative array, whereas in Experiments 1 and 2 these data slightly shifted to the positive. This variability suggests that these deviations are random.

The results showed that episodic memory effects cannot account for the transfer of adaptation effects to pictures of familiar faces that were not shown during adaptation. Rather, it seems that our adaptation procedure had a lasting effect on both individual and general face representations, and that a time period of several days is not sufficient to fully compensate for these changes in cognitive organization. In fact, it is plausible that the integration of extreme visual stimulation into pre-existing face representations has a strong and long-lasting effect that cannot be averaged out quickly even by a large number of brief encounters with normal faces. The interplay between adaptation and re-adaptation mechanisms as a function of adaptation and re-adaptation intensity as well as the temporal aspects involved have yet to be studied.

\section{General Discussion}

Our data from three experiments show that exposure to configurally distorted faces caused a bias in the perception of the veridical face away from the distorted Gestalt. On average, participants who inspected strongly compressed versions of a face selected slightly compressed faces when asked to pick the veridical version of the target face. An analogous pattern occurred for participants who adapted to strongly extended versions, but was not found in the subjects of the control condition who adapted to original, that is, veridical, versions. This indicates that representations of familiar faces are very flexible and can be subject to quick and efficient adjustments in response to new perceptual information.

To induce strong and long-lasting aftereffects, a prolonged adaptation procedure was used during which participants were exposed to strongly distorted (though still recognizable) pictures of familiar faces for approximately $25 \mathrm{~min}$. The adaptation procedure was realized by employing a time-sensitive gender-decision task, which has already been proven to be an efficient means of generating pronounced adaptation effects (e.g., Carbon, Strobach, et al., 2007). In this specific task, participants were exposed to faces presented in different positions on the screen for variable durations, which meant that the participant had to maintain constant attention. Many traditional studies investigating face adaptation have shown that much shorter adaptation durations are sufficient to induce reliable short-term effects for unfamiliar faces (e.g., 1 to

Table 2

Mean Selections in Experiment 3

\begin{tabular}{lccc}
\hline Distortion & $M$ & $S E$ & \multicolumn{1}{c}{$95 \% \mathrm{CI}$} \\
\hline-5 & -.273 & .076 & $-.435,-.112$ \\
Original & -.096 & .076 & $-.258, .065$ \\
+5 & .306 & .076 & $.144, .467$ \\
\hline
\end{tabular}

Note. Experiment 3 was for the novel condition only (24-hr delay).
16 s; Leopold et al., 2005; Rhodes et al., 2007) that, when tested, were reported to decay exponentially in the course of several hundred milliseconds (Leopold et al., 2001; Leopold et al., 2005; Rhodes et al., 2007). The procedure used in this study seems to have accessed the more deeply rooted memory traces of highly familiar faces, enabling us to affect the participants' perception in the long run.

In the present study, participants were tested 5 min (Experiment 1), $24 \mathrm{hr}$ (Experiment 3), or 7 days (Experiment 2) after adaptation for face veridicality of highly familiar faces. Adaptation effects have been found in all experiments and on all levels of transfer, that is, the condition picture (using pictorially identical pictures for adaptation and test), the condition identity (conducting the test using different images of the same person), and the condition novel (testing new faces). The long delay between the two sessionsespecially the 1-week period-would suggest that adaptation cannot be explained solely by low-level retinotopic adaptation. Rather, it seems that adaptation altered participants' representations of individual faces, as well as affected more abstract face representations (i.e., the representations of broader face subpopulations and/or a general face norm), which is in line with normbased theories of higher level aftereffects (e.g., Carbon, Strobach, et al., 2007b; Leopold et al., 2001; Rhodes \& Jeffery, 2006). Regardless of the transfer levels, face veridicality aftereffects seem to decrease over the course of time and were stronger in the 5-min condition than in the 1 -week condition. This decline may be explained by a passive decay that occurs automatically if the extreme visual experiences that caused the effect are not repeated. Alternatively, this may be explained by more active re-adaptation mechanisms in response to various encounters with normal-shaped faces during the interval between the two experimental sessions. To our knowledge, the exact nature of such recalibration processes has not yet been investigated. Studies focusing on adaptation decay as a function of (a) adaptation duration, (b) delay, and (c) experiences of re-adaptation after different temporal intervals as well as different intensity levels would be useful for further understanding the underlying principles of ongoing perceptual adjustments. This might also be interesting from a methodological perspective on adaptation research, because the findings revealed by long-term adaptation studies imply the possibility of considerable carryover effects between different conditions in common experimental designs (McKone et al., 2005).

The significant 1-week transfer effect is of particular interest because we expected it to vanish over the course of 7 days as a result of participants' repeated interactions with normal faces in everyday life. There are several possible explanations for why it did not vanish. First, a period of 1 week may be not long enough to fully compensate for the integration of extreme visual experiences into face representations, whether through passive decay or active re-adaptation to natural configurations or, more likely, a combination of the two. Second, in terms of a hierarchical model of norm-based face coding, it is conceivable that adaptation does not operate independently on the various levels of abstraction due to the significant overlap between the different face norms. A single picture of a familiar face belongs to an individual prototype (identity-specific representation), but also contributes to the face norm that is abstracted from the entire face pool (general face representation). Adaptation-induced alterations in one of these representations would thus automatically lead to changes on other levels, too. The 
transfer effect therefore could be interpreted as a simple byproduct of the multiple, identity-specific and long-term aftereffects. Third, we should discuss potential context effects that could have affected our results. Experiment 3 was designed to account for episodic memory effects that might have biased participants' test face selections in the transfer condition without any representational basis, still yielding a reliable effect. To fully rule out these possible confounds, it might be useful in future studies to manipulate the settings of the two experimental sessions more rigorously by changing the perceptual context (e.g., test room, lighting conditions, time of day, etc.). Nevertheless, please note that we do not consider such contextual effects to be a valid alternative explanation for all the face aftereffects that were found in the present study. It is unlikely that a general and unspecific context effect could account for the fine variations in the size of the adaptation effects between the three levels of transfer as well as their hierarchic order.

A more specific alternative explanation is based on the encoding specificity principle: "What is stored is determined by what is perceived and how it is encoded, and what is stored determines what retrieval cues are effective in providing access to what is stored" (Tulving \& Thomson, 1973, p. 353). Leder and Carbon (2005) found that the specific size of the presentation of a face (whole vs. partbased presentation) is essential for the encoding and, consequently, the further processing of the face. They found that the compatibility of the presentation size between learning and test phase is essential for high performance in recognizing faces, thus extending the classical holistic effect by Tanaka and Farah (1993), where this was only shown for holistically learned faces. With reference to the present study, we could claim that the more similar the encoding (adaptation) and the decoding (test), the stronger the encoding specificity effect (adaptation effect). We would in fact find a compatible pattern if we were to qualify the novel transfer condition as a condition, where the degree of compatibility between encoding and decoding is still relatively high. Regarding the novel transfer condition, we could argue that the mere configuration (first-order relations) of specific famous faces in the encoding phase is compatible with the mere configuration of the novel faces in the decoding phase. Although we cannot exclude this alternative explanation, we argue in favor of an adaptation mechanism that at least partially operates on the basis of memory representations. The reason for this is twofold: First, we need an adaptive mechanism that integrates the latest information into existing memory structures. Such a mechanism is very helpful in enabling us to react specifically, quickly, and accurately to any target object. The generated representation most probably provides by definition the best fit with the outside world as it is "updated." Second, although the effects of the novel transfer condition in all experiments are generally compatible with the encoding specificity principle, this explanation needs further ad hoc hypotheses, as different stimuli are encoded than retrieved in this condition. The adaptation explanation can solve this issue by suggesting that adaptation mechanisms are in action in the whole face space. Deviated, odd, or distinctive faces result in changes to the overall face space. The face space adjusts to new visual inputs and the full variety of experienced faces. The encoding specificity explanation would have to account for the effects in terms of a more generalized encoding specificity that functions, for instance, on the basis of the processing of general configurations, which in our view is less probable.

\section{Conclusions}

We believe that the study of sustained face aftereffects is relevant to our theoretical understanding of the ongoing adaptive processes in the neuro-cognitive system and the dynamics of higher level mechanisms involved in the perception of complex stimuli. The longevity of our effects points toward multiple timescales in the operation of adaptation mechanisms. Moreover, longterm face aftereffects can be explained more readily by representational rather than merely perceptual models of adaptation, supporting theories of prototype-referenced face coding. In future studies, the intensity of adaptation, the delay, and the frequency of inspected faces between adaptation and test should be varied across even longer time ranges, which may provide important information for understanding the link between short-term and transient neuro-cognitive plasticity and the formation of long-term changes associated with learning and memory.

\section{References}

Bales, J. F., \& Follansbee, G. L. (1935). The after-effect of the perception of curved lines. Journal of Experimental Psychology, 18, 499-503.

Bestelmeyer, P. E. G., Jones, B. C., DeBruine, L. M., Little, A. C., Perrett, D. I., Schneider, A., ... Conway, C. A. (2008). Sex-contingent face aftereffects depend on perceptual category rather than structural encoding. Cognition, 107, 353-365.

Bruce, V. (1994). Stability from variation-The case of face recognition. Quarterly Journal of Experimental Psychology A: Human Experimental Psychology, 47, 5-28.

Carbon, C. C. (2008). Famous faces as icons. About the illusion of being an expert in the recognition of famous faces. Perception, 37, 801-806.

Carbon, C. C., Ditye, T., \& Leder, H. (2007). When style matters-Art specific adaptation effects [Abstract]. Perception Abstracts, 36.

Carbon, C. C., \& Leder, H. (2005). Face adaptation: Changing stable representations of familiar faces within minutes? Advances in Experimental Psychology, 1(1), 1-7.

Carbon, C. C., \& Leder, H. (2006). The Mona Lisa effect: Is "our" Lisa fame or fake? Perception, 35, 411-414.

Carbon, C. C., Strobach, T., Langton, S. R. H., Harsányi, G., Leder, H., \& Kovacs, G. (2007). Adaptation effects of highly familiar faces: Immediate and long lasting. Memory and Cognition, 35, 1966-1976.

Cohen, J. D., MacWhinney, B., Flatt, M., \& Provost, J. (1993). PsyScope: A new graphic interactive environment for designing psychology experiments. Behavior Research Methods Instruments \& Computers, 25, 257-271.

Faulkner, T. F., Rhodes, G., Palermo, R., Pellicano, E., \& Ferguson, D. (2002). Recognizing the un-real McCoy: Priming and the modularity of face recognition. Psychonomic Bulletin \& Review, 9, 327-334.

Gibson, J. J., \& Radner, M. (1937). Adaptation, after-effect and contrast in the perception of tilted lines. I. Quantitative studies. Journal of Experimental Psychology, 20, 453-467.

Hammer, E. R. (1949). Temporal factors in figural after-effects. American Journal of Psychology, 62, 337-354.

Hepler, N. (1968). Color-A motion-contingent aftereffect. Science, 162(3851), 376-377.

Hershenson, M. (1989). Duration, time constant, and decay of the linear motion aftereffect as a function of inspection duration. Perception \& Psychophysics, 45, 251-257.

Hurlbert, A. (2001). Trading faces. Nature Neuroscience, 4(1), 3-5.

Jaquet, E., \& Rhodes, G. (2008). Face aftereffects indicate dissociable, but 
not distinct, coding of male and female faces. Journal of Experimental Psychology: Human Perception and Performance, 34, 101-112.

Jenkins, R., Beaver, J. D., \& Calder, A. J. (2006). I thought you were looking at me-Direction-specific aftereffects in gaze perception. Psychological Science, 17, 506-513.

Jiang, F., Blanz, V., \& O'Toole, A. J. (2006). Probing the visual representation of faces with adaptation-A view from the other side of the mean. Psychological Science, 17, 493-500.

Jiang, F., Blanz, V., \& O'Toole, A. J. (2009). Three-dimensional information in face representations revealed by identity aftereffects. Psychological Science, 20, 318-325.

Jones, P. D., \& Holding, D. H. (1975). Extremely long-term persistence of McCollough effect. Journal of Experimental Psychology: Human Perception and Performance, 1, 323-327.

Kloth, N., \& Schweinberger, S. R. (2008). The temporal decay of eye gaze adaptation effects. Journal of Vision, 8(11), 1-11.

Koehler, W. (1944). Figural aftereffects. An investigation of visual processes. Proceedings of the American Philosophical Society, 88, 269357.

Kovacs, G., Cziraki, C., Vidnyanszky, Z., Schweinberger, S. R., \& Greenlee, M. W. (2008). Position-specific and position-invariant face aftereffects reflect the adaptation of different cortical areas. NeuroImage, 43, $156-164$.

Kovacs, G., Zimmer, M., Banko, E., Harza, I., Antal, A., \& Vidnyanszky, Z. (2006). Electrophysiological correlates of visual adaptation to faces and body parts in humans. Cerebral Cortex, 16, 742-753.

Kovacs, G., Zimmer, M., Harza, I., \& Vidnyanszky, Z. (2007). Adaptation duration affects the spatial selectivity of facial aftereffects. Vision Research, 47, 3141-3149.

Krauskopf, J. (1954). The magnitude of figural after-effects as a function of the duration of the test-period. American Journal of Psychology, 67, $684-690$

Leder, H., \& Carbon, C. C. (2005). When context hinders! Learn-test compatibility in face recognition. The Quarterly Journal of Experimental Psychology A: Human Experimental Psychology, 58, 235-250.

Leopold, D. A., \& Bondar, I. (2005). Adaptation to complex visual patterns in humans and monkeys. In C. W. G. Clifford \& G. Rhodes (Eds.), Fitting the mind to the world: Adaptation and after-effects in high-level vision (1st ed., pp. 189-211). New York, NY: Oxford University Press.

Leopold, D. A., O’Toole, A. J., Vetter, T., \& Blanz, V. (2001). Prototypereferenced shape encoding revealed by high-level after effects. Nature Neuroscience, 4, 89-94.

Leopold, D. A., Rhodes, G., Muller, K. M., \& Jeffery, L. (2005). The dynamics of visual adaptation to faces. Proceedings of the Royal Society B: Biological Sciences, 272(1566), 897-904.

Little, A. C., DeBruine, L. M., \& Jones, B. C. (2005). Sex-contingent face after-effects suggest distinct neural populations code male and female faces. Proceedings of the Royal Society B: Biological Sciences, 272(1578), 2283-2287.

Masland, R. H. (1969). Visual motion perception-Experimental modification. Science, 165(3895), 819-821.

McCollough, C. (1965). Color adaptation of edge-detectors in human visual system. Science, 149(3688), 1115-1116.

McKone, E., Edwards, M., Robbins, R., \& Anderson, R. (2005). The stickiness of face adaptation aftereffects [Abstract]. Journal of Vision, 5, 822 .

Neitz, J., Carroll, J., Yamauchi, Y., Neitz, M., \& Williams, D. R. (2002). Color perception is mediated by a plastic neural mechanism that is adjustable in adults. Neuron, 35, 783-792.

Rakover, S. S. (2002). Featural vs. configurational information in faces: A conceptual and empirical analysis. British Journal of Psychology, 93, $1-30$.

Rhodes, G., Brennan, S., \& Carey, S. (1987). Identification and ratings of
caricatures-Implications for mental representations of faces. Cognitive Psychology, 19, 473-497.

Rhodes, G., \& Jeffery, L. (2006). Adaptive norm-based coding of facial identity. Vision Research, 46, 2977-2987.

Rhodes, G., Jeffery, L., Clifford, C. W. G., \& Leopold, D. A. (2007). The timecourse of higher-level face aftereffects. Vision Research, 47, 22912296.

Rhodes, G., Jeffery, L., Watson, T.-L., Clifford, C.-W. G., \& Nakayama, K. (2003). Fitting the mind to the world: Face adaptation and attractiveness aftereffects. Psychological Science, 14, 558-566.

Rhodes, G., Jeffery, L., Watson, T. L., Jaquet, E., Winkler, C., \& Clifford, C. W. G. (2004). Orientation-contingent face aftereffects and implications for face-coding mechanisms. Current Biology, 14, 2119-2123.

Rhodes, G., Robbins, R., Jaquet, E., McKone, E., Jeffery, L., \& Clifford, C. W. G. (2005). Adaptation and face perception-How aftereffects implicate norm-based coding of faces. In C. W. G. Clifford \& G. Rhodes (Eds.), Fitting the mind to the world-Adaptation and after-effects in high-level vision (1st ed., pp. 213-240). New York, NY: Oxford University Press.

Robbins, R., McKone, E., \& Edwards, M. (2007). Aftereffects for face attributes with different natural variability: Adapter position effects and neural models. Journal of Experimental Psychology: Human Perception and Performance, 33, 570-592.

Schweinberger, S. R., Kloth, N., \& Jenkins, R. (2007). Are you looking at me? Neural correlates of gaze adaptation. NeuroReport, 18, 693-696.

Suzuki, S., \& Cavanagh, P. (1998). A shape-contrast effect for briefly presented stimuli. Journal of Experimental Psychology: Human Perception and Performance, 24, 1315-1341.

Tanaka, J. W., \& Farah, M. J. (1993). Parts and wholes in face recognition. Quarterly Journal of Experimental Psychology A: Human Experimental Psychology, 46, 225-245.

Taylor, M. J., Arsalidou, M., Bayless, S. J., Morris, D., Evans, J. W., \& Barbeau, E. J. (2009). Neural correlates of personally familiar faces: Parents, partner and own faces. Human Brain Mapping, 30, 2008-2020.

Tulving, E., \& Thomson, D. M. (1973). Encoding specificity and retrieval processes in episodic memory. Psychological Review, 80, 352-373.

Valentine, T. (1991). A unified account of the effects of distinctiveness, inversion, and race in face recognition. Quarterly Journal of Experimental Psychology A: Human Experimental Psychology, 43, 161-204.

Valentine, T. (2001). Face-space models of face recognition. In M. J. Wenger \& J. T. Townsend (Eds.), Computational, geometric, and process perspectives on facial cognition: Contexts and challenges ( $\mathrm{pp}$. 83-113). Mahwah, NJ: Erlbaum.

Valentine, T., \& Endo, M. (1992). Towards an exemplar model of face processing-The effects of race and distinctiveness. Quarterly Journal of Experimental Psychology A: Human Experimental Psychology, 44, 671-703.

Vernon, M. D. (1934). The perception of inclined lines. British Journal of Psychology, 25, 186-196.

Vul, E., Krizay, E., \& MacLeod, D. I. A. (2008). The McCollough effect reflects permanent and transient adaptation in early visual cortex. Journal of Vision, 8(12), 1-12.

Wade, N. J., Swanston, M. T., \& Deweert, C. M. M. (1993). On interocular transfer of motion aftereffects. Perception, 22, 1365-1380.

Wade, N. J., \& Verstraten, F. A. J. (2005). Accommodating the past: A selective history of adaptation. In C. W. G. Clifford \& G. Rhodes (Eds.), Fitting the mind to the world: Adaptation and after-effects in high-level vision (1st ed., pp. 83-101). New York, NY: Oxford University Press.

Webster, M. A., Kaping, D., Mizokami, Y., \& Duhamel, P. (2004). Adaptation to natural facial categories. Nature, 428(6982), 557-561.

Webster, M. A., \& MacLin, O. H. (1999). Figural aftereffects in the perception of faces. Psychonomic Bulletin \& Review, 6, 647-653. 
Webster, M. A., \& Miyahara, E. (1997). Contrast adaptation and the spatial structure of natural images. Journal of the Optical Society of America A: Optics Image Science and Vision, 14, 2355-2366.

Webster, M. A., Werner, J. S., \& Field, D. J. (2005). Adaptation and the phenomenology of perception. In C. W. G. Clifford \& G. Rhodes (Eds.), Fitting the mind to the world-Adaptation and after-effects in high-level vision (pp. 241-277). New York, NY: Oxford University Press.

Yamashita, J. A., Hardy, J. L., De Valois, K. K., \& Webster, M. A. (2005). Stimulus selectivity of figural aftereffects for faces. Journal of Experimental Psychology: Human Perception and Performance, $31,420-437$.

Zhao, L., \& Chubb, C. (2001). The size-tuning of the face-distortion after-effect. Vision Research, 41, 2979-2994.

Received July 14, 2009

Revision received January 31, 2010

Accepted February 8, 2010

\section{ORDER FORM}

Start my 2011 subscription to the Journal of Experimental

Psychology: Human Perception and Performance ISSN: 0096-1523

\$167.00 APA MEMBER/AFFILIATE

$\$ 392.00$ INDIVIDUAL NONMEMBER

$\$ 1,148.00$ INSTITUTION

In DC and MD add $6 \%$ sales tax

TOTAL AMOUNT DUE

$\$$

Subscription orders must be prepaid. Subscriptions are on a calendar year basis only. Allow 4-6 weeks for delivery of the first issue. Call for international subscription rates.

SEND THIS ORDER FORM TO

American Psychological Association

Subscriptions

750 First Street, NE

Washington, DC 20002-4242

AMERICAN PSYCHOLOGICAL

ASSOCIATION

Call 800-374-2721 or 202-336-5600

Fax 202-336-5568 :TDD/TTY 202-336-6123

For subscription information,

e-mail: subscriptions@apa.org $\square$ Check enclosed (make payable to APA)

Charge my: $\square$ Visa $\square$ MasterCard $\square$ American Express

Cardholder Name

Card No. _ Exp. Date

Signature (Required for Charge)

\section{Billing Address}

Street

City

State

Zip

Daytime Phone

E-mail

\section{Mail To}

Name

Address

City State

Zip

APA Member \# 